\title{
Facile formation of giant elastin-like polypeptide vesicles as synthetic cells
}

\author{
Bineet Sharma ${ }^{1}$, Yutao $\mathrm{Ma}^{2}$, Andrew L Ferguson², Allen P Liu ${ }^{1,3,4,5}$ \\ ${ }^{1}$ Department of Mechanical Engineering, University of Michigan, Ann Arbor, Michigan \\ 48109, USA \\ ${ }^{2}$ Pritzker School of Molecular Engineering, University of Chicago, Chicago, Illinois 60637, \\ USA \\ ${ }^{3}$ Department of Biomedical Engineering, University of Michigan, Ann Arbor, Michigan \\ 48109, USA \\ ${ }^{4}$ Cellular and Molecular Biology Program, University of Michigan, Ann Arbor, Michigan \\ 48105, USA \\ ${ }^{5}$ Department of Biophysics, University of Michigan, Ann Arbor, Michigan 48105, USA
}

\section{Corresponding author:}

A.P.L.: allenliu@umich.edu; 2350 Hayward Street, University of Michigan, Ann Arbor, Michigan 48109. Tel: +1 734-764-7719. 


\begin{abstract}
Creating a suitable compartment for synthetic cells has led the exploration of different cell chassis materials from phospholipids to polymer to protein-polymer conjugates. Currently, the majority of cell-like compartments are made of lipid molecules as the resulting membrane resembles that of a natural cell. However, cell-sized lipid vesicles are prone to physical and chemical stresses and can be unstable in hosting biochemical reactions within. Recently, peptide vesicles that are more robust and stable were developed as a new chassis material for synthetic cells. Here we demonstrate the facile and robust generation of giant peptide vesicles made of elastin-like polypeptides (ELPs) by using an emulsion transfer method. We show that these peptide vesicles can stably encapsulate molecules and can host cell-free expression reactions. We also demonstrate membrane incorporation of another amphiphilic ELP into existing peptide vesicles. Since ELPs are genetically encoded, the approaches presented here provide exciting opportunities to engineer synthetic cell membranes.
\end{abstract}




\section{Introduction}

Synthetic cells are cell-like compartments in the form of membrane vesicles. ${ }^{1,2} \mathrm{~A}$ synthetic cell provides a framework where internally encapsulated materials are protected from the external aqueous environment with minimum leakage. Ideally, the protecting boundary should be mechanically strong and semi-permeable for nutrients and gaseous exchange. Various amphiphilic molecules have been employed to create a robust cell-like system, including phospholipids, ${ }^{3-5}$ fatty acids, ${ }^{6}$ polymers, ${ }^{7}$ double emulsion, ${ }^{8,9}$ and a hybrid of protein-polymer ${ }^{10}$ or lipid-polymer. ${ }^{11}$ Phospholipid bilayer vesicles hold the most resemblance to natural cell membrane and have been employed as drug delivery vehicles. ${ }^{12,13}$ The application of these materials in applied synthetic cell research is impacted by their high susceptibility towards physical stresses like osmotic shock and mechanical deformation and chemical stress like oxidation in response to $\mathrm{pH}$ change. Polymersomes with improved mechanical strength were introduced, ${ }^{14,15}$ and the use of synthetic polymer in building synthetic cell continues to grow. ${ }^{16}$

Recently, a genetically encoded elastin-like polypeptide (ELP) was introduced in a synthetic cell model by Pirzer and co-workers and they demonstrated cell-free synthesis of ELP in growing ELP vesicles. ${ }^{17,18}$ ELP has a basic structure of a repeating pentapeptide entity Val-Pro-Gly-X-Gly, where the guest residue $X$ can be any natural amino acid except proline. ELPs exhibit a reversible lower critical solution temperature (LCST) behavior and changes its phase above a transition temperature $\left(T_{t}\right)$ due to selective assembly of hydrophobic blocks. ELPs remain soluble when temperature is lowered below $T_{t .}{ }^{19}$ Based on this feature, ELPs can be purified by inverse transition cycling method. ${ }^{20}$ ELPs are quite versatile and have been exploited for applications in drug delivery and tissue engineering. ${ }^{21,22}$ Although ELPs have been shown to form $\sim 50 \mathrm{~nm}$ unilamellar vesicles, ${ }^{23}$ there are few examples of generating cell-sized ELP vesicles. This is in part due to the propensity for ELPs to form an array of micellar structures. We hypothesize that amphiphilic ELPs may be able to form bilayer vesicles when presented a template.

Here we demonstrate a robust method for generating giant ELP vesicles as synthetic cells by using an emulsion transfer method originally developed by Pautot et al. ${ }^{24}$ with modification. The peptide bilayer was subsequently confirmed by incorporation of a membrane labelling dye and a fluorescently labeled lipid. ELP vesicles are compatible with bacterial cell-free expression (CFE) and we show that they can host CFE reactions to 
express proteins of interest. Finally, we demonstrate membrane incorporation of another amphiphilic ELP into existing ELP vesicles. The robust generation of ELP vesicles that support CFE reactions significantly advances synthetic cell engineering as it provides a new genetically encoded approach for engineering membrane without relying on lipidproducing enzymes.

\section{Results}

\section{Formation of giant peptide vesicle and peptide bilayer}

We start with an amphiphilic ELP, denoted as S48I48, that comprises of 48 repeats of pentapeptide (VPGXG) blocks of serine and isoleucine as the guest residue for the hydrophilic and hydrophobic domains, respectively (Figure 1A). S48I48 has a total of 483 amino acids with MW of $39.8 \mathrm{kDa}$, with each hydrophilic and hydrophobic domain comprises of 240 amino acids. $\$ 48148$ displays partial transition at $27^{\circ} \mathrm{C}$ due to selfassembly of isoleucine blocks and bulk phase transition at $75^{\circ} \mathrm{C}$ due to aggregation of serine blocks. ${ }^{25}$ S48148 was expressed and purified from $E$. coli by three cycles of inverse transition cycling at $37^{\circ} \mathrm{C}$ and the purity of a lyophilized ELP was confirmed by SDS-PAGE (Supplementary Figure 1); the stock ELP solution is stored in a chloroform:methanol mixture.

There exist several methods for generating giant vesicles and many of them are based on generating large water-in-oil emulsion droplets that are stabilized by amphiphilic molecules $^{16}$. A monolayer of amphiphilic molecules can self-assemble at the oil-water interphase between two bulk phases. Centrifugation of the emulsion droplets across the monolayer-stabilized interface generates bilayer vesicles. We applied this emulsion transfer method to generate giant ELP vesicles (Figure 1B). The S48I48 was dissolved in an oil mixture of mineral oil:silicone oil (1:4) and a trace amount of fluorescent lipid, NBD$\mathrm{PE}$, was used to label peptide vesicles. Giant S48148 vesicles recovered from the water phase had a range of sizes from 3 to $>50 \mu \mathrm{m}$ with an average size of $15.1 \mu \mathrm{m}$ (Figure 1C).

To rule out the possibility that NBD-PE was somehow driving the formation of giant peptide vesicles, we made unlabeled S48I48 vesicles and asked if a membrane labeling dye can partition into the bilayer and label the peptide membrane. Following incubation of 
a cell membrane labelling dye, Vibrant $\mathrm{DiO}$, we observed robust labeling of S48I48 vesicles (Figure 1D). When we performed emulsion transfer with the same concentration of NBD-PE used without including S48148, no vesicles were formed (data not shown). Together, these results demonstrate that amphiphilic ELPs can robustly form peptide vesicles and are compatible with emulsion transfer techniques.

A distinct advantage of an ELP-based compartment is that these peptides can be genetically encoded and thus we can leverage the power of cell-free protein expression ${ }^{26}$ to synthesize ELPs in situ. However, we do not expect the expressed ELP to selfassemble into cell-sized vesicles. It is known that in the presence of hydrophilic silica microspheres that small lipid vesicles can rupture on them forming supported lipid bilayers. We next investigated whether nano-ELP vesicles could form supported peptide bilayers using the supported bilayers with excess membrane reservoir (SUPER) template that has been used to study membrane-localized processes and membrane protein reconstitution. ${ }^{27,28}$ Following CFE of S48/48, we incubated the CFE reaction with $5 \mu \mathrm{m}$ silica beads and a trace amount of NBD-PE at $37^{\circ} \mathrm{C}$ in a high salt solution. This is above $T_{t}$ for S48148 and we suspect under high salt condition, S48148 micelles/nanovesicles fuse with the silica beads. A uniform labeling of peptide bilayer was observed on the silica beads as shown in Figure 1E. A solution of silica beads without cell-free expressed S48I48 and incubated with NBD-PE under the same condition did not yield any labeled membrane on the beads, again signifying that NBD-PE alone cannot form bilayer at the concentration used.

Experimental determination of the bilayer thickness is challenging due to resolution limitations of conventional optical microscopy. Instead, we conducted coarse-grained molecular dynamics simulations of the bilayer to predict its thickness and internal molecular structure. We present in Figure 1F a snapshot of the fully relaxed bilayer at 300 $\mathrm{K}$ and 1 bar together with the partial density profiles for the (VPGSG) 48 segment, (VPGIG) ${ }_{48}$ segment and water across the bilayer. We observed the bilayer to quickly adopt a well-defined structure and morphology that remained stable over the course of the $1.8 \mu \mathrm{s}$ production run, indicating that, consistent with experimental observations, the bilayer morphology is a stable morphology. The upper $\sim 2.5 \mathrm{~nm}$ of each leaflet of the bilayer defines a transition region within which we observe limited incursion of water into the bilayer. Our calculations predict the thickness of the each of the two distal hydrophilic S48 
regions to be $(31.7 \pm 0.1) \mathrm{nm}$, the central hydrophobic 148 region to be $(67.9 \pm 0.1) \mathrm{nm}$, and the transition region between $\mathrm{S} 48$ and 148 regions to be $3.0 \pm 0.1 \mathrm{~nm}$, for a total bilayer thickness of $(134.4 \pm 0.1) \mathrm{nm}$.

\section{Encapsulation of dye and proteins in peptide vesicles}

Armed with a robust strategy to generate giant peptide vesicles, we next examined the capacity of these peptide vesicles for encapsulation of small molecules and proteins as this is an important feature of a synthetic cell model. As one would expect from an emulsion transfer method, encapsulation of a small molecule dye (e.g., Rhodamine-B) was robust in S48148 vesicles (Figure 2A), and we did not detect observable leakage of the encapsulated dye from overnight incubation at room temperature (data not shown).

We next examined the compatibility of bacterial CFE reactions with our newly developed peptide vesicle system and tested two scenarios: i) encapsulation of CFE reaction following protein expression, and ii) in situ expression of proteins from encapsulation of CFE reaction. Using GFP as a reporter, our home-made E. coli CFE robustly produced GPF over 4 hours (Figure 2B). Using this solution as the inner solution for making single emulsion droplets in S48I48-containing oil phase, GFP can be detected in the S48I48 peptide vesicles (Figure 2C), as one would expect. Similarly, cell-free expressed GFP is detected in S48148 peptide vesicles when the CFE reaction was directly encapsulated and incubated (Figure 2D). These results demonstrate that giant $\$ 48 \mid 48$ ELP peptide vesicles can host CFE reactions similar to giant lipid bilayer vesicles.

\section{Membrane incorporation of cell-free expressed amphiphilic ELP}

The growth of the vesicle, considered to be an important feature of synthetic cell development, can be achieved by addition of membrane ingredients ${ }^{29-31}$ or by using lipid modifying enzymes either introduced as a purified protein ${ }^{32}$ or by cell-free expression ${ }^{33}$. For ELP vesicles, a recent study reported by Frank et al. ${ }^{34}$ showed the growth of peptide vesicles is driven by a combination of osmotic-driven changes due to CFE reactions and fusion of membrane components fed from the outside. Here we sought to directly incorporate cell-free expressed ELP into pre-formed ELP vesicles. For this purpose, we 
selected another ELP RQ-F that has a free lysine at position 30 so that we could use fluorescently labeled lysine to visualize the synthesized ELP. RQ-F is an abbreviation of $\left[(V P G R G)_{5}(V P G Q G)_{5}\right]_{2}(V P G F G)_{20}$ which is an amphiphilic ELP with arginine $(R)$ and glutamine $(\mathrm{Q})$ blocks constituting the 20 pentapeptide repeat of the hydrophilic block and phenylalanine $(\mathrm{F})$ constituting the 20 pentapeptide repeat of the hydrophobic block (note that the lysine residue is not denoted in the molecular formula). RQ-F can be fluorescently labelled in situ by incorporating FluoroTect Green Lys during CFE and we hypothesize that encapsulation Green Lys labeled RQ-F (RQ-F ${ }^{\text {GreenLys }}$ ) can be incorporated into the ELP vesicle membrane (Figure 3A). In a batch CFE reaction, we find that $R Q-F^{\text {GreenLys }}$ was expressed very well (Figure 3B). When cell-free expressed $R Q-F^{\text {GreenLys }}$ was encapsulated without purification inside S48I48 peptide vesicles under an iso-osmotic condition and incubated at RT for 2 hours, we observed clear membrane incorporation of RQ-F ${ }^{\text {GreenLys }}$ at several locations that are concurrent with visible deformation of the peptide vesicle (Figure 3C). The incorporation of $R Q-F^{G r e e n L y s}$ did not appear to be uniform. In distinct contrast, we did not find labeling of the membrane with only Green Lys alone in CFE reactions without expressing $R Q-F$.

\section{Discussion}

Using an emulsion transfer method, we report micron-sized peptide vesicles made of amphiphilic S48I48 ELP. ELPs represent alternative chassis materials for synthetic cell construction for several reasons, including: (i) their stability in harsh physical and chemical conditions; (ii) inexpensive and relatively easy to synthesize; (iii) easy to purify with high yield; (iv) easy to maneuver their polarity by introducing different guest residues. Unlike lipid vesicles, the S48148 peptide vesicles appear to have a thick membrane, from brightfield images, due of its large hydrophobic and hydrophilic blocks each constituting 240 amino acids. To the best of our knowledge, peptide vesicles comprised of S48I48 is the largest polypeptide sequence reported to form giant peptide compartments.

The first demonstration of using an amphiphilic ELP to create self-assembled vesicular structures as reaction compartments for synthetic cells utilized a glass bead swelling method that yielded peptide vesicles of $\sim 200 \mathrm{~nm}$ in diameter. ${ }^{17}$ Subsequently, the same group devised a solvent evaporation method ${ }^{34}$ that consists of first dissolving lyophilized 
ELPs in tetrahydrofuran followed by adding the inner solution to be encapsulated with agitation. Evaporation of tetrahydrofuran followed by addition of outer solution resulted in two populations of ELP vesicles, ones that are tens of nanometer in diameter and ones that are over $1 \mu \mathrm{m}$ in size. Since the emulsion transfer method is commonly used for lipid vesicle encapsulation, its direct applicability to make peptide vesicles eliminates the use of harsh organic solvents like tetrahydrofuran. Since the size of emulsion droplets can be controlled by using microfluidics, an emulsion transfer method described here would allow for creation of synthetic cells with homogeneous sizes. As with other emulsion-based methods for vesicle generation, we cannot rule out the possibility that there remains residual oil within the ELP bilayer.

Although we have demonstrated the facile and robust formation of giant peptide vesicles using only S48148 as our model ELP, we believe this approach is generally applicable to other ELP sequences. The key conceptual advance in our approach is that ELPs can be templated on aqueous droplets or micron-sized solid supports in ways that are similar when using amphiphilic lipid molecules. As ELPs are genetically encodable and can be expressed by CFE, there is the potential for carrying out high throughput screening of ELP sequences with varying guest residues and block lengths for their ability to form peptide vesicles. This could greatly expand the discovery of additional self-assembling ELPs that can be used as synthetic cell membrane. Our demonstration that a different cellfree expressed ELP can incorporate into existing ELP vesicles made of another ELP presents opportunities for generating multi-functional peptide vesicles by leveraging peptide sequences that can be appended to provide additional functionalities.

\section{Conclusion}

In summary, this study presents an emulsion transfer method as a facile approach for generating giant peptide vesicles and highlights the use of amphiphilic peptides as a chassis material for synthetic cell research. Using S48I48 as our model ELP, we show the robust generation of giant peptide vesicles that can be labeled with fluorescently labeled lipids or membrane dye molecules. These giant peptide vesicles can host bacterial CFE reactions and express proteins of interest. We further demonstrate that ELPs expressed by CFE can incorporate into existing an ELP membrane. These highly stable peptide 
vesicles could serve as a robust platform to advance research in bottom-up synthetic biology.

\section{Acknowledgement}

S48I48 ELP was a kind gift from Andrew MacKay, University of Southern California, USA (Addgene plasmid no. 68394), RQ-F ELP was a kind gift from Tobias Pirzer, Technical University Munich, Germany. This work is supported by the National Science Foundation under Grant Nos. DMR-1939354 (APL) and DMR-1939463 (ALF).

\section{Conflict of interest}

ALF is a co-founder and consultant of Evozyne, LLC and a co-author of US Provisional Patents 62/853,919 and 62/900,420 and International Patent Applications PCT/US2020/035206 and PCT/US20/50466. 


\section{Materials and Methods}

\section{Materials}

S48I48 ELP was a kind gift from Andrew MacKay, University of Southern California, USA (Addgene plasmid no. 68394), RQF ELP was a kind gift from Tobias Pirzer, Technical University Munich, Germany. All the chemicals were procured from SigmaAldrich (St Louis, MI) unless otherwise specified. Vibrant DiO was purchased from Invitrogen. NBD-PE and Rho-PE were purchased from Avanti Polar Lipids. Mineral oil was purchased from Acros Organics. BLR(DE3) Competent cells-Novagen were procured from Millipore Sigma. Terrific Broth powder was purchased from Thermo Fisher. Slide-ALyzer $^{\text {TM }}$ MINI Dialysis Device, 10K MWCO, $2 \mathrm{~mL}$ was purchased from Thermo Fisher Scientific. FluoroTect ${ }^{\mathrm{TM}}$ Green $_{\mathrm{Lys}}$ in vitro Translation Labeling System was purchased from Promega. Silica beads were procured from Bangs Laboratories (Fishers, IN).

\section{Expression and purification}

The plasmid pET-25b(+) encoding S48I48 ELP was transformed into Escherichia coli $B L R(D E 3)$ strain that is suitable for expressing recombinant proteins with repetitive sequences. A starter culture was inoculated and grown overnight ( 14 hours) in $50 \mathrm{~mL}$ Terrific Broth supplemented with $100 \mu \mathrm{g} / \mathrm{mL}$ ampicillin at $37^{\circ} \mathrm{C}$ at $200 \mathrm{rpm}$. 1-2 volume \% of the starter culture was then scaled up to $1 \mathrm{~L}$ culture and grown for 24 hours at $37^{\circ} \mathrm{C}$ at $200 \mathrm{rpm}$ in the presence of ampicillin. ELP was synthesized by the leaky expression of the T7 RNA polymerase without using IPTG.

Cell pellets were collected in a pre-weighed centrifuge tube by centrifugation at $6,000 \times$ $\mathrm{g}$ for 10 minutes at $4{ }^{\circ} \mathrm{C}$ and resuspended in $30 \mathrm{~mL}$ PBS. The resuspended cells were washed with phosphate buffer saline (PBS) by centrifugation at $4,000 \times \mathrm{g}$ for 20 minutes at $4^{\circ} \mathrm{C}$ to obtain the cell pellet mass. For each gram of cell pellet, $2 \mathrm{ml}$ of lysis buffer was used for cell lysis by sonication. Lysis buffer contains PBS with $1 \mathrm{mg} / \mathrm{mL}$ lysozyme, $1 \mathrm{mM}$ phenylmethylsulfonyl fluoride (PMSF), $1 \mathrm{mM}$ benzamidine, and $0.5 \mathrm{U}$ of DNase I. Cells were sonicated using Branson Sonifier Sound Enclosure with settings of output power of 5, duty cycle $50 \%$, and 10 -second sonication with 20-second pause time for 6 minutes, followed by incubation on ice for 10 minutes. For complete lysis, the steps were repeated twice. For the precipitation of genomic DNA, $2 \mathrm{~mL}$ of $10 \% \mathrm{v} / \mathrm{v}$ polyethyleneimine (PEI) was added to the cell lysate. Supernatant containing ELP was collected after aliquoting the 
lysate in $1.5 \mathrm{~mL}$ Eppendorf tubes, followed by centrifugation at $16,000 \times \mathrm{g}$ for 10 minutes at $4^{\circ} \mathrm{C}$. At least three rounds of inverse transition cycling (ITC) $)^{20}$ were performed to purify S48I48. This method harnesses the phase transition temperature of ELP for its purification. S48I48 ELP exhibits two transition temperatures, one at $27^{\circ} \mathrm{C}$ (due to the isoleucine blocks) and another at $75^{\circ} \mathrm{C}$ (due to the serine blocks). ${ }^{25}$ The lower transition temperature was used for the purification of ELP. Briefly, in hot-spin cycle of ITC, the ELP solution was heated above transition temperature (here $37^{\circ} \mathrm{C}$ ) followed by dropwise addition of $5 \mathrm{M} \mathrm{NaCl}$ until the solution becomes cloudy. Immediately, the pellet was collected by centrifugation at $16,000 \times \mathrm{g}$ for $10 \mathrm{~min}$. For a cold-spin cycle, pellet containing ELP was re-suspended in cold PBS and incubated at ice for 10 minutes, followed by supernatant collection at $16,000 \times \mathrm{g}$ for $10 \mathrm{~min}$. ELP transitions from pellet (hot-spin) to supernatant (cold-spin) in single round of ITC. In the final round of ITC, supernatant was then dialyzed using Slide-A-Lyzer MINI device by following the manufacturer's protocol to remove excess salt and undesired proteins. Lyophilized sample was stored at $-20^{\circ} \mathrm{C}$. Purity of ELP was determined by standard SDS-PAGE.

\section{Peptide vesicles preparation and labeling}

Peptide vesicles were prepared by using the emulsion transfer method. A stock solution of $\sim 135 \mu \mathrm{M}(5.44 \mathrm{mg} / \mathrm{mL})$ concentration of ELP was prepared in a mixture of chloroform:methanol $(2: 7)$ and stored at $-20^{\circ} \mathrm{C}$ in a sealed glass vial until use. $99.9 \mathrm{~mol} \%$ of S48I48 $(10 \mu \mathrm{M})$ along with $0.1 \mathrm{~mol} \%$ NBD-PE were dissolved in an oil phase containing a mixture of mineral oil:silicone oil (1:4). For optical phase contrast, a combination of 200 $\mathrm{mM}$ sucrose and $200 \mathrm{mM}$ glucose were used as inner and outer aqueous solutions, respectively. About $130 \mu$ of water-in-oil emulsion was generated by vortexing $100 \mu \mathrm{L}$ of oil mixture containing ELP followed by addition of $30 \mu \mathrm{L}$ of sucrose solution. An interface was established by first adding $200 \mu$ glucose solution in a $1.5 \mathrm{ml}$ Eppendorf tube, followed by $400 \mu \mathrm{l}$ of ELP-containing oil phase and left undisturbed for at least $15 \mathrm{~min}$. The water-in-oil emulsion was then carefully transferred to the oil phase and centrifuged at $10,000 \times \mathrm{g}$ for 10 minutes at room temperature (RT). The oil phase was carefully removed without disturbing the giant peptide vesicles at the bottom. Vesicles were retrieved by pipetting and observed under confocal fluorescence microscopy. 
Giant peptide vesicles were labelled by a cell membrane labelling dye, Vibrant DiO. Briefly, DiO was mixed with peptide vesicles at a ratio of 1:200 following the manufacturer's protocol and observed under a fluorescence microscope at least after 1 hour of incubation at RT.

\section{ELP-coated beads}

ELP-coated silica beads were prepared using SUPER template method described by Pucadyil et $\mathrm{al}^{27}$ with slight modification. Cell-free expressed S48I48 was incubated for 10 min at $37^{\circ} \mathrm{C}$ in the presence of PBS and $1 \mathrm{M} \mathrm{NaCl}$, followed by addition of $5 \mu \mathrm{l}$ of $5 \mu \mathrm{m}$ silica bead solution $\left(6.4 \times 10^{6}\right.$ beads $\left./ \mathrm{ml}\right)$ with $1 \mu \mathrm{L}$ NBD-PE $(1 \mathrm{mg} / \mathrm{mL})$ and incubated at RT for 30 minutes with intermittent tapping. Beads were washed twice with PBS by centrifugation at $500 \times \mathrm{g}$ for 3 minutes and observed under a fluorescence microscope.

\section{Cell-free GFP expression}

Plasmid containing T7p14deGFP was expressed using homemade CFE components following the protocol by Sun et al. ${ }^{35}$ Briefly, a $10 \mu \mathrm{L}$ cell-free reaction was prepared by using S30 extract and protein synthesis buffer along with T7 RNA polymerase (21.25 $\mathrm{ng} / \mu \mathrm{l})$ RNase inhibitor, plasmid encoded GFP (1 nM) and water. All the components were vigorously mixed and incubated at $30{ }^{\circ} \mathrm{C}$ for 4 hours. Fluorescence intensity with end-point kinetic was recorded using a Biotek fluorescence plate reader. CFE-expressed GFP was then encapsulated in peptide vesicles using emulsion transfer method in an iso-osmotic condition.

For in situ expression of GFP in peptide vesicles, the CFE components were assembled along with a GFP-expressing plasmid and encapsulated as the inner solution as described in previous paragraph. Vesicles were then incubated at $30^{\circ} \mathrm{C}$ for 4 hours and the expressed protein was observed under microscope.

\section{Membrane incorporation of ELP RQ-F}

In vitro synthesis of ELP RQ-F was carried out using in-house CFE reaction for 4 hours at $30^{\circ} \mathrm{C}$, supplemented with FluoroTect Green Lys, by following the manufacturer's protocol. This labelling system incorporates the lysine-labeled with BODIPY®-FL into ELP 
$R Q-F$ sequence, making it fluorescent. $R Q-F^{\text {GreenLys }}$ was encapsulated inside ELP S48I48 vesicles and incubated at RT for at least 2 hours for membrane incorporation.

\section{Microscopy}

All the peptide vesicles images were acquired using an oil immersion 60×/1.4 NA PlanApochromat objective lens mounted on an Olympus IX-81 inverted fluorescence microscope (Olympus Corporation, Tokyo, Japan) equipped with a CSU-X1 spinning disc confocal head (Yokogawa Electric Corporation, Tokyo, Japan), AOTF-controlled solidstate lasers (Andor Technology, Belfast, UK), and an iXON3 EMCCD camera (Andor Technology, Belfast, UK). Fluorescence images were captured using MetaMorph software (Molecular Devices, San Jose, CA) with laser excitation at $488 \mathrm{~nm}$ for GFP, DiO, and Green Lys and $561 \mathrm{~nm}$ for Rho-PE and rhodamine. All the image analysis was performed using NIH-ImageJ.

\section{Molecular dynamics simulation}

The all-atom structure of (VPGSG) ${ }_{48}(\mathrm{VPGIG})_{48}$ chain was prepared using PyMOL software. ${ }^{36}$ The all-atom structure was then coarse-grained using Martinize.py script ${ }^{37}$ and all interactions were modeled by Martini v2.2 force field. ${ }^{37-39}$ The (VPGSG) ${ }_{48}(\mathrm{VPGIG})_{48}$ bilayer membrane was initialized by first placing the (VPGSG) ${ }_{48}(\mathrm{VPGIG})_{48}$ chains on a 10by-10 grid where the centers of mass (COM) of neighboring chains were separated by 0.9 $\mathrm{nm}$. This monolayer of peptide chains was then flipped across the $\mathrm{x}-\mathrm{y}$ plane to obtain a bilayer. The solvent-exposed N-termini of the hydrophilic (VPGSG) 48 blocks were set to have +1 charge while the buried C-termini of the hydrophobic (VPGIG) 48 blocks were set to be neutral. Coarse-grained water molecules and $\mathrm{Cl}^{-}$ions were then added above and below the bilayer using the PACKMOL package ${ }^{40}$. The initial system size was $9 \mathrm{~nm} \times 9 \mathrm{~nm}$ $\times 261 \mathrm{~nm}$.

Molecular dynamics simulations were conducted using the Gromacs 2019.3 simulation suite. ${ }^{41}$ The initial system configuration was first energy minimized using the steepest descent algorithm to eliminate all forces in excess of $1000 \mathrm{~kJ} / \mathrm{mol} . \mathrm{nm}$. Initial atomic velocities were then sampled from a Maxwell-Boltzmann distribution at $300 \mathrm{~K}$ and the system was subjected to $40 \mathrm{~ns}$ of NVT equilibration at $300 \mathrm{~K}$. We then performed a 60 ns NPT equilibration at $300 \mathrm{~K}$ and 1 bar. Finally, we conducted a $1.8 \mu$ s NPT production 
run at $300 \mathrm{~K}$ and 1 bar. We verified that the equilibration period was sufficiently long to relax the bilayer to its equilibrium structure by verifying that the thickness, structure, and density profiles of the bilayer remained stable over the course of the $1.8 \mu$ s production run. Simulation parameters were selected following best practices for coarse-grained simulations using the Martini model. ${ }^{42}$ Numerical integration of the classical equations of motion was conducted using the leapfrog algorithm ${ }^{43}$ with a $20 \mathrm{fs}$ time step. The temperature was maintained using a stochastic velocity rescaling thermostat with a time constant of $1 \mathrm{ps}^{44}$ During the NPT equilibration the pressure was controlled using the Berendsen barostat ${ }^{45}$ with a time constant of $12 \mathrm{ps}$, whereas during the NPT production run the pressure was controlled using the Parrinello-Rahman barostat ${ }^{46}$ with a time constant of 12 ps. The bond constraints were handled by LINCS method ${ }^{47}$ and electrostatics was handled by reaction-field method. ${ }^{48}$ 


\section{Figure captions}

Figure 1: Generation of giant peptide vesicles. (A) Amphiphilic ELP with 48 repeats of serine and isoleucine blocks constitute hydrophilic and hydrophobic domains, respectively. (B) Schematic representation of emulsion transfer method for giant peptide vesicles formation. Firstly, ELP S48I48 is dissolved in the oil mixture mineral oil:silicone oil (1:4) with NBD-PE dye. Oil-water interface was created for the directed assembly of amphiphilic polypeptide molecules (step 1). An emulsion containing water-in-oil droplets with an ELP monolayer is transferred to the oil phase (step 2). Giant peptide vesicles are collected by centrifugation at 10,000 $\times \mathrm{g}$ for 10 minutes and removal of oil (step 3 and 4). (C) Peptide vesicle size distribution with average size $15.1 \mu \mathrm{m}$; inset image of a single peptide vesicle labelled with NBD-PE. $N_{\text {vesicles }}=112$. (D) Schematic illustrating peptide vesicle labeling with Vibrant DiO dye after its preparation and incubation and representative brightfield and fluorescence images of a labeled $\$ 48148$ peptide vesicle at least after 1-hour labeling. (E) Schematic illustrating cell-free expressed S48|48 forming peptide bilayer on $5 \mu \mathrm{m}$ silica beads using the SUPER template procedure. Beads were washed twice before observation. Fluorescence images of silica beads with or without cell-free expressed S48I48. Scale bars are $10 \mu \mathrm{m}$. (F) The terminal snapshot from a coarse-grained molecular dynamics simulation of the S48I48 bilayer together with the (VPGSG) 48 segment, $(\mathrm{VPGIG})_{48}$ segment and water partial density profiles calculated across the bilayer. The bilayer thickness is predicted to be $(134.4 \pm 0.1) \mathrm{nm}$.

Figure 2: Peptide vesicles as a chassis for synthetic cell. (A) Encapsulation of small molecules like rhodamine dye in a S48148 vesicle. (B) Bulk expression of GFP by an $E$. coli CFE reaction incubated for 4 hours at $30^{\circ} \mathrm{C}$ and measured on a fluorescence plate reader. (C) Schematic illustrating cell-free expressed protein (GFP) encapsulated inside peptide vesicles generated by emulsion transfer method. (D) Fluorescence images of cellfree expressed GFP encapsulated in a S48I48 vesicle. (E) Schematic illustrating encapsulation of CFE reaction to express GFP. Brightfield and fluorescence images of S48/48 vesicle after $4 \mathrm{hr}$ of expression at $30^{\circ} \mathrm{C}$. Scale bars are $10 \mu \mathrm{m}$. 
Figure 3: Incorporation of ELP RQ-F ${ }^{\text {GreenLys }}$ into the peptide membrane. (A) Fluorescent labelling of ELP RQ-F by incorporating green lysine during CFE by supplementing the reaction with FluoroTect Green Lys. Labelled RQ-F was encapsulated inside peptide vesicles and incubated for at least 2 hours at room temperature. (B) SDS-PAGE showed the fluorescently labelled $R Q-F$ with a trail of unbound reagent. $(C)$ Brightfield and fluorescence images of the ELP S48I48 peptide vesicle encapsulating RQ-F ${ }^{\text {GreenLys }}$. (D) Brightfield and fluorescence images of the ELP S48I48 peptide vesicle encapsulating CFE reaction with green lysine but without RQ-F DNA. Scale bars are $10 \mu \mathrm{m}$. 


\section{References}

1. Szostak, J. W., Bartel, D. P. \& Luisi, P. L. Synthesizing life. Nature vol. 409 387-390 (2001).

2. Stano, P. Is research on "synthetic cells" moving to the next level? Life 9, 3 (2019).

3. Noireaux, V. \& Libchaber, A. A vesicle bioreactor as a step toward an artificial cell assembly. Proc. Natl. Acad. Sci. U. S. A. 101, 17669-17674 (2004).

4. Nishimura, K. et al. Cell-free protein synthesis inside giant unilamellar vesicles analyzed by flow cytometry. Langmuir 28, 8426-32 (2012).

5. Majumder, S. et al. Cell-sized mechanosensitive and biosensing compartment programmed with DNA. Chem. Commun. 53, 7349-7352 (2017).

6. Chen, I. A., Roberts, R. W. \& Szostak, J. W. The emergence of competition between model protocells. Science (80-. ). 305, 1474-1476 (2004).

7. Martino, C. et al. Protein Expression, Aggregation, and Triggered Release from Polymersomes as Artificial Cell-like Structures. Angew. Chemie 124, 6522-6526 (2012).

8. Caschera, F., Lee, J. W., Ho, K. K. Y., Liu, A. P. \& Jewett, M. C. Cell-free compartmentalized protein synthesis inside double emulsion templated liposomes with in vitro synthesized and assembled ribosomes. Chem. Commun. (Camb). 52, 5467-9 (2016).

9. Ho, K. K. Y., Lee, J. W., Durand, G., Majumder, S. \& Liu, A. P. Protein aggregation with poly(vinyl) alcohol surfactant reduces double emulsionencapsulated mammalian cell-free expression. PLoS One 12, (2017).

10. Huang, X. et al. Interfacial assembly of protein-polymer nano-conjugates into stimulus-responsive biomimetic protocells. Nat. Commun. 4, 1-9 (2013).

11. Jacobs, M. L., Boyd, M. A. \& Kamat, N. P. Diblock copolymers enhance folding of a mechanosensitive membrane protein during cell-free expression. Proc. Natl. Acad. Sci. 116, 4031-4036 (2019).

12. Shah, S., Dhawan, V., Holm, R., Nagarsenker, M. S. \& Perrie, Y. Liposomes: Advancements and innovation in the manufacturing process. Advanced Drug Delivery Reviews vols 154-155 102-122 (2020).

13. Elkhoury, K. et al. Engineering Smart Targeting Nanovesicles and Their Combination with Hydrogels for Controlled Drug Delivery. Pharmaceutics 12, 849 (2020).

14. Discher, B. M. et al. Polymersomes: Tough vesicles made from diblock copolymers. Science (80-. ). 284, 1143-1146 (1999).

15. Rideau, E., Dimova, R., Schwille, P., Wurm, F. R. \& Landfester, K. Liposomes and polymersomes: a comparative review towards cell mimicking. Chem. Soc. Rev. 47, 8572-8610 (2018). 
16. Groaz, A. et al. Engineering spatiotemporal organization and dynamics in synthetic cells. WIREs Nanomedicine and Nanobiotechnology e1685 (2020) doi:10.1002/wnan.1685.

17. Vogele, K. et al. Towards synthetic cells using peptide-based reaction compartments. Nat. Commun. 9, 3862 (2018).

18. Schreiber, A., Huber, M. C. \& Schiller, S. M. Prebiotic Protocell Model Based on Dynamic Protein Membranes Accommodating Anabolic Reactions. Langmuir 35 , 9593-9610 (2019).

19. Urry, D. W. Physical chemistry of biological free energy transduction as demonstrated by elastic protein-based polymers. Journal of Physical Chemistry $B$ vol. 101 11007-11028 (1997).

20. Meyer, D. E. \& Chilkoti, A. Purification of recombinant proteins by fusion with thermally-responsive polypeptides. Nat. Biotechnol. 17, 1112-1115 (1999).

21. Saha, S., Banskota, S., Roberts, S., Kirmani, N. \& Chilkoti, A. Engineering the Architecture of Elastin-Like Polypeptides: From Unimers to Hierarchical SelfAssembly. Adv. Ther. 3, 1900164 (2020).

22. Varanko, A. K., Su, J. C. \& Chilkoti, A. Elastin-Like Polypeptides for Biomedical Applications. Annu. Rev. Biomed. Eng. 22, 343-369 (2020).

23. Pastuszka, M. K. et al. An amphipathic alpha-helical peptide from apolipoprotein A1 stabilizes protein polymer vesicles. J. Control. Release 191, 15-23 (2014).

24. Pautot, S., Frisken, B. J. \& Weitz, D. A. Production of unilamellar vesicles using an inverted emulsion. Langmuir 19, 2870-2879 (2003).

25. Shah, M. et al. Biodegradation of elastin-like polypeptide nanoparticles. Protein Sci. 21, 743-750 (2012).

26. Noireaux, V. \& Liu, A. P. The New Age of Cell-Free Biology. Annu. Rev. Biomed. Eng. 22, 51-77 (2020).

27. Pucadyil, T. J. \& Schmid, S. L. Supported Bilayers with Excess Membrane Reservoir: A Template for Reconstituting Membrane Budding and Fission. Biophys. J. 99, 517-525, (2010).

28. Majumder, S., Willey, P. T., DeNies, M. S., Liu, A. P. \& Luxton, G. A synthetic biology platform for the reconstitution and mechanistic dissection of LINC complex assembly. J. Cell Sci. 132, (2019).

29. Hanczyc, M. M., Fujikawa, S. M. \& Szostak, J. W. Experimental Models of Primitive Cellular Compartments: Encapsulation, Growth, and Division. Science (80-. ). 302 , 618-622 (2003).

30. Zhu, T. F. \& Szostak, J. W. Coupled growth and division of model protocell membranes. J. Am. Chem. Soc. 131, 5705-5713 (2009).

31. Tsuji, G., Fujii, S., Sunami, T. \& Yomo, T. Sustainable proliferation of liposomes 
compatible with inner RNA replication. Proc. Natl. Acad. Sci. U. S. A. 113, 590-595 (2016).

32. Bhattacharya, A., Brea, R. J., Niederholtmeyer, H. \& Devaraj, N. K. A minimal biochemical route towards de novo formation of synthetic phospholipid membranes. Nat. Commun. 10, 300 (2019).

33. Blanken, D., Foschepoth, D., Serrão, A. C. \& Danelon, C. Genetically controlled membrane synthesis in liposomes. doi:10.1038/s41467-020-17863-5.

34. Frank, T., Vogele, K., Dupin, A., Simmel, F. C. \& Pirzer, T. Growth of Giant Peptide Vesicles Driven by Compartmentalized Transcription-Translation Activity. Chem. - A Eur. J. 26, 1 - 6 (2020).

35. Sun, Z. Z. et al. Protocols for implementing an Escherichia coli based TX-TL cellfree expression system for synthetic biology. J. Vis. Exp. 50762 (2013) doi:10.3791/50762.

36. Schrödinger LLC. The PyMOL Molecular Graphics System, Version 1.8. (2015).

37. de Jong, D. H. et al. Improved Parameters for the Martini Coarse-Grained Protein Force Field. J. Chem. Theory Comput. 9, 687-697 (2013).

38. Marrink, S. J., Risselada, H. J., Yefimov, S., Tieleman, D. P. \& de Vries, A. H. The MARTINI Force Field: Coarse Grained Model for Biomolecular Simulations. J. Phys. Chem. B 111, 7812-7824 (2007).

39. Monticelli, L. et al. The MARTINI Coarse-Grained Force Field: Extension to Proteins. J. Chem. Theory Comput. 4, 819-834 (2008).

40. Martinez, L., Andrade, R., Birgin, E. G. \& Martínez, J. M. PACKMOL: A package for building initial configurations for molecular dynamics simulations. J. Comput. Chem. 30, 2157-2164 (2009).

41. Abraham, M. J. et al. GROMACS: High performance molecular simulations through multi-level parallelism from laptops to supercomputers. SoftwareX 1-2, 19-25 (2015).

42. De Jong, D. H., Baoukina, S., Ingólfsson, H. I. \& Marrink, S. J. Martini straight: Boosting performance using a shorter cutoff and GPUs. Comput. Phys. Commun. 199, 1-7 (2016).

43. Hockney, R. W. \& Eastwood, J. W. Computer Simulation Using Particles. Computer Simulation Using Particles (IOP Publishing Ltd, 1988). doi:10.1887/0852743920.

44. Bussi, G., Donadio, D. \& Parrinello, M. Canonical sampling through velocity rescaling. J. Chem. Phys. 126, 014101 (2007).

45. Berendsen, H. J. C., Postma, J. P. M., Van Gunsteren, W. F., Dinola, A. \& Haak, J. R. Molecular dynamics with coupling to an external bath. J. Chem. Phys. 81, 36843690 (1984).

46. Parrinello, M. \& Rahman, A. Polymorphic transitions in single crystals: A new 
molecular dynamics method. J. Appl. Phys. 52, 7182-7190 (1981).

47. Hess, B., Bekker, H., Berendsen, H. J. C. \& Fraaije, J. G. E. M. LINCS: A Linear Constraint Solver for molecular simulations. J. Comput. Chem. 18, 1463-1472 (1997).

48. Watts, R. O. Monte Carlo studies of liquid water. Mol. Phys. 28, 1069-1083 (1974). 